This document is confidential and is proprietary to the American Chemical Society and its authors. Do not copy or disclose without written permission. If you have received this item in error, notify the sender and delete all copies.

\title{
Active nanorheology with plasmonics
}

\begin{tabular}{|r|l|}
\hline Journal: & Nano Letters \\
\hline Manuscript ID & nl-2016-014049.R1 \\
\hline Manuscript Type: & Communication \\
\hline Date Submitted by the Author: & 26-Jun-2016 \\
\hline Complete List of Authors: & $\begin{array}{l}\text { Jeong, Hyeon-Ho; Max-Planck-Institut fur Intelligente Systeme, } \\
\text { Mark, Andrew; Max Planck Institute for Intelligent Systems, } \\
\text { Lee, Tung Chun; University College London, Institute for Materials } \\
\text { Discovery } \\
\text { Alarcón-Correa, Mariana ; Max-Planck-Institut fur Intelligente Systeme } \\
\text { Eslami, Sahand; Max-Planck-Institut fur Intelligente Systeme } \\
\text { Qiu, Tian; Max-Planck-Institut fur Intelligente Systeme } \\
\text { Gibbs, John; Northern Arizona University, Physics and Astronomy } \\
\text { Fischer, Peer; Max Planck Institute for Intelligent Systems, }\end{array}$ \\
\hline
\end{tabular}

SCHOLARONE ${ }^{\mathrm{m}}$

Manuscripts 


\section{Active nanorheology with plasmonics}

Hyeon-Ho Jeong ${ }^{1,2, *}$, Andrew G. Mark ${ }^{1, *}$, Tung-Chun Lee ${ }^{1,3}$, Mariana Alarcón-Correa ${ }^{1,4}$, Sahand Eslami ${ }^{1,4}$,Tian Qiu ${ }^{1,5}$, John G. Gibbs ${ }^{1,6}$, and Peer Fischer ${ }^{1,4, *}$

${ }^{1}$ Max Planck Institute for Intelligent Systems, Heisenbergstrasse 3, 70569 Stuttgart, Germany

${ }^{2}$ Institute of Materials, École Polytechnique Fédérale de Lausanne (EPFL), CH-1015

Lausanne, Switzerland

${ }^{3}$ UCL Institute for Materials Discovery and Department of Chemistry, University College

London, Christopher Ingold Building, 20 Gordon Street, London WC1H 0AJ, United

Kingdom

${ }^{4}$ Institute for Physical Chemistry, University of Stuttgart, Pfaffenwaldring 55, 70569 Stuttgart, Germany

${ }^{5}$ Institute of Bioengineering, École Polytechnique Fédérale de Lausanne (EPFL), CH-1015

Lausanne, Switzerland

${ }^{6}$ Department of Physics and Astronomy, Northern Arizona University, S. San Francisco

Street, Flagstaff, Arizona 86011, USA

These authors contributed equally 


\begin{abstract}
Nanoplasmonic systems are valued for their strong optical response and their small size. Most plasmonic sensors and systems to date have been rigid and passive. However, rendering these structures dynamic opens new possibilities for applications. Here we demonstrate that dynamic plasmonic nanoparticles can be used as mechanical sensors to selectively probe the rheological properties of a fluid in situ at the nanoscale and in microscopic volumes. We fabricate chiral magneto-plasmonic nanocolloids that can be actuated by an external magnetic field, which in turn allows for the direct and fast modulation of their distinct optical response. The method is robust and allows nanorheological measurements with a mechanical sensitivity of $\sim 0.1 \mathrm{cP}$, even in strongly absorbing fluids with an optical density of up to OD $3(\sim 0.1 \%$ light transmittance) and in the presence of scatterers (e.g. 50\% v/v red blood cells).
\end{abstract}

\title{
KEYWORDS.
}

Magneto-plasmonics, chiral plasmonics, chiroptical switch, nanorheology

The resonant optical field enhancement in plasmonic nanostructures is of interest in research disciplines ranging from sensing to energy conversion, and is the basis for modern advances in metamaterials and the associated capabilities in shaping electromagnetic fields ${ }^{1-3}$. The scope of potential applications of nanoplasmonic structures is greatly extended by tailoring the nanostructure, for instance to shift the resonance frequency while keeping the overall size of the nanoparticle small ${ }^{4-7}$, or by incorporating diverse functionalities, such as magnetic $^{8}$, chiral ${ }^{9}$, and electrical ${ }^{10}$. Recent fabrication advances have also allowed the programmatic growth of nanoparticles that lack mirror symmetry ${ }^{11-15}$. Such chiral nanoparticles mimic their molecular counterparts by exhibiting optical activity, but with 


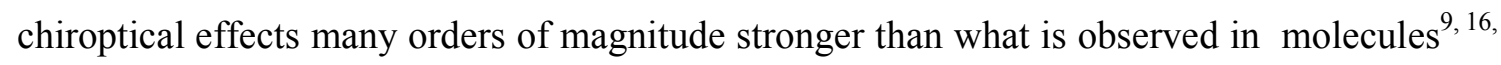

17 . These multifunctional nanoparticles promise new capabilities in sensing ${ }^{18,19}$.

One particularly challenging application is the measurement of rheological properties in complex fluids. Such systems contain a mixture of multiple phases; for instance, many biological fluids contain solids consisting of isolated microparticles or a network of macromolecules suspended in a fluid phase ${ }^{20}$. Because of the solid phase, macroscopic rheological measurements will generally show non-Newtonian viscoelastic behavior even if the liquid phase is a simple Newtonian fluid ${ }^{21}$. For instance, the viscosity of blood plasma is a crucial indicator for clinical diagnoses ${ }^{22,23}$, but cannot be determined in whole blood due to the presence of leukocytes $(10-15 \mu \mathrm{m})$ and erythrocytes $(6-8 \mu \mathrm{m})^{24,25}$. Furthermore, the solid phase in complex fluids, such as blood cells, contributes to absorption and scattering which complicate optical measurements.

By combining multiple materials and shape control, we here introduce chiral magnetoplasmonic structures that can be actuated in solution. Since the chiroptical spectrum depends on the alignment of the chiral structure (Figure 1a), we can achieve chiroptical switching and exploit it for nanorheological measurements using picomolar probe concentrations. The scheme works by using the modulated chiroptical signal to measure the phase lag between the applied field and the particle's orientation and thereby determine the torque applied to the particles (Figure 1c). The chiral shape permits background-free differential absorption measurements even in whole blood, where conventional plasmonic absorption measurements would be impossible due to the large background optical extinction (optical density 3). Because the active plasmonic nanostructures are much smaller than the suspended microparticles (cells), they probe only the fluid phase - in the case of blood, the blood plasma (Figure 1b) - and unlike macroscale instruments are insensitive to the solid phase even at very high volume fractions. Such an in situ observations of the fluid viscosity would not be 
possible with a conventional rheometer or viscometer, which typically requires much larger volumes of the target fluid $(>10 \mathrm{~mL})$.

Active plasmonic systems have recently been developed by controlling either the electromagnetic radiation applied to the metallic nanostructure ${ }^{26-29}$ or the dynamics of the nanostructures ${ }^{30-34}$. However, to the best of our knowledge, there are no plasmonic systems that can be switched repeatedly at high frequency and that satisfy the minimum requirements for in situ active nanorheology in a complex fluid: (1) a distinguishable optical contrast despite strong extinction (and no bleaching), (2) small size relative to the suspended microparticles (e.g. erythrocytes) $)^{35,36}$, and (3) high nanoparticle monodispersity for a precise hydrodynamic response ${ }^{37}$. These criteria are satisfied by the $\sim 170 \mathrm{~nm}$ magneto-plasmonic nanohelices shown in Figure 2. Briefly, their fabrication is based on a published combination of block-copolymer micelle nanolithography $(\mathrm{BCML})^{38}$ and physical vapor glancing angle deposition $(\mathrm{GLAD})^{39}$, which we term nanoGLAD ${ }^{12}$. BCML is used to form a quasi-hexagonal array of $\mathrm{Au}$ nanoparticles with diameter $12 \pm 2 \mathrm{~nm}$ and an interparticle distance $\sim 100 \mathrm{~nm}$ on a silicon wafer. Vapors of elemental $\mathrm{Au}$ and Fe were then co-deposited in a 1:1.5 ratio onto the nano-patterned substrate using a dual e-beam system to generate an array of $\mathrm{Au}-\mathrm{Fe}$ nanohelices. Sonication in a fluid readily detaches the helices from the wafer to yield the nanocolloidal suspension used in our experiments, Figure 2a-b. Details of the geometry and material composition are given in Supporting Information Note S1. Magnetic actuation of the nanoparticles is possible due to the presence of Fe (Figure 2c). Superconducting quantum interference device (SQUID) magnetometry was carried out on the array of Au-Fe nanohelices when they were still attached to the wafer. As expected, the helices display ferromagnetic behavior, having a coercive field of $29.5 \mathrm{mT}$ and a remanence of $1.2 \times 10^{-14}$ emu per single helix, as seen in Figure 2d. 
In dilute suspensions, where the inter-particle separations are large, the nanoparticles will independently undergo Brownian motion and thus maintain random orientations in space. In this isotropic state, the optical response is simply given by the average response across all possible nanoparticle orientations. The optical properties of a suspension containing the nanohelices in the unaligned, isotropic state are summarized in Figure 2e. The extinction spectrum, measured by UV-VIS-NIR spectrometry, shows a wide featureless spectrum typical of mixing a lossy material (Fe) with the plasmonic metal $\mathrm{Au}^{40,41}$. However, the power of circular dichroism (CD) spectroscopy is apparent in the strong, sharp chiroptical response of the Au-Fe nanohelix metafluid. At a peak wavelength of $690 \mathrm{~nm}$, it exhibits a molar $\mathrm{CD}=$ $\sim 14 \times 10^{9}{ }^{\circ} \cdot \mathrm{M}^{-1} \cdot \mathrm{cm}^{-1}$, stronger than that observed for other chiral assembled plasmonic systems $^{11,42-44}$. Complex fluids, such as blood, whose rheology we set out to measure generally show no measurable chiroptical response of their own in this spectral region ${ }^{45}$. Thus the chiroptical spectrum of chiral nanoparticles dispersed in such media is not affected by the background extinction from the fluid and can be 'seen', even in the presence of absorbers and strong scatterers.

Application of an external field causes alignment of the magnetic particles due to the natural magnetic anisotropy along the long axis of the helix ${ }^{46}$. In the static regime, a weakly ferromagnetic $\mathrm{Au}-\mathrm{Fe}$ nanohelix will orient so that its magnetic moment $\mathbf{m}$, and hence its body, lies parallel to the magnetic field, B. However it retains rotational freedom to spin about its body-axes. The optical response of an asymmetric particle depends on its orientation relative to the wave vector $\mathbf{k}$ of the incoming beam. For linearly polarized light the optical response will be a function of two angles: $\theta_{B}$ between $\mathbf{B}$ and $\mathbf{k}$ (assuming that $\mathbf{B} \| \mathbf{m}$ ) and the angle between $\mathbf{B}$ and the plane of polarization. However, since circularly polarized light possesses no plane of polarization, the chiroptical response measured by $\mathrm{CD}$ spectroscopy will be a function of only the single parameter $\theta_{B}$. Thus, in the chiral metafluid, the magnetic 
modulation effectively switches the isotropic medium into a uniaxial one, where the principal axis is defined by the magnetic field direction.

In our experiments, the orientation of the Au-Fe nanohelices in solution was modulated by an external magnetic field generated by a gradient-free 3D Helmholtz coil (See Figure S1). Based on the magnetic moment per particle, the minimum field strength to achieve orientational alignment is expected to be only $\sim 0.4 \mathrm{mT}$ at room temperature (See Supporting Information Note S2). Thus, the experiments were conducted with magnetic fields $|\mathbf{B}|>0.4$ mT (approx. 10 times Earth's magnetic field) to ensure alignment of the nanocolloids. Higher fields can exert stronger torques. Since a gradient-free field was used, the translational diffusion of the particles was unaffected, even as the rotational diffusion was suppressed. Real-time direct observation of the colloidal nanohelices was performed with dark-field optical microscopy (see Supporting Information Note S3). The image panels in Figure 3a show consecutive dark-field images of a single Au-Fe nanohelix captured during continuous in-plane rotation driven by the applied magnetic field $\left(\mathbf{B}=5 \mathrm{mT}\right.$ with $f_{B}=1 \mathrm{~Hz}$, in a $50 \%$ glycerol-water mixture). The features of the helix are too small to be resolved so it appears as an elongated ellipse; crucially, the ellipse axis, which corresponds to the helix axis, rotates in alignment with the magnetic field direction. Thus, the magnetic moment of the nanohelix allows an external magnetic field of this strength to control its orientation.

The effect of the nanocolloids' orientation on the chiroptical response was evaluated through $\mathrm{CD}$ spectra acquired at various magnetic field orientations. (See Figure $\mathrm{S} 1 \mathrm{~b}$ ). At $\theta_{B}=$ $0^{\circ}$, the maximum molar $\mathrm{CD}$ of the Au-Fe nanohelices was $\sim 36 \times 10^{9}{ }^{\mathrm{o}} \cdot \mathrm{M}^{-1} \cdot \mathrm{cm}^{-1}$ (See Supporting Information Note S4). We characterize the effect through the differential circular dichroism $\Delta \mathrm{CD}=\mathrm{CD}\left(\theta_{B}\right)-<\mathrm{CD}>$ to emphasize differences between $\operatorname{CD}\left(\theta_{B}\right)$, the signal with the magnetic field oriented at $\theta_{B}$, and $<\mathrm{CD}>$ the isotropic case with $\mathbf{B}=0$. The spectra in Figure $3 \mathrm{a}$ show the experimental $\triangle \mathrm{CD}$ spectra in response to applied magnetic fields at 
different orientations (See Figure S10 for full spectra). They show a clear modulation of the signal, with a symmetry that reflects the underlying symmetries of the particles and the imposed constraints. First, the spectra are invariant under $\pi$-rotation of $\theta_{B}$ due to the $C_{2}$ symmetry of the helix. Second, the helices' unconstrained degree of freedom about their axis means that $\operatorname{CD}\left(\theta_{B}\right)=\operatorname{CD}\left(-\theta_{B}\right)$. Figure $3 b$ shows the differential $\mathrm{CD}$ spectra displayed as $2 \mathrm{D}$ maps in terms of $\lambda$ and $\theta_{B}$. These results are in excellent agreement with numerical simulations using the discrete dipole approximation (See Figure 3c, and Supporting Information Note S5 for details of numerical calculation method).

Figure $3 \mathrm{~d}$ shows $\Delta \mathrm{CD}$ profiles at wavelengths $\lambda=360 \mathrm{~nm}$ and $1,000 \mathrm{~nm}$, where the $\Delta C D$ responses were minimal and maximal at $\theta_{B}=0^{\circ}$ respectively, acquired with static magnetic fields at specified angles. For the long wavelength spectral curve at $\theta_{B}=0^{\circ}$, the $\mathrm{CD}$ is over 200 mdeg higher than in the isotropic state. With increasing $\theta_{B}$ the differential CD diminishes until it reaches a minimum at $\theta_{B}=90^{\circ}$. The $\lambda=360 \mathrm{~nm}$ curve follows the opposite trend. The two curves cross each other, and the $\Delta \mathrm{CD}=0^{\circ}$ axis at an angle of $\sim 50^{\circ}$. The lower panel of Figure $3 \mathrm{~d}$ shows the corresponding results from numerical simulations. High frequency modulation of the magnetic field leads to rapid, synchronous and reversible modulation of the differential CD. Figure 3e shows the differential $\mathrm{CD}$ at 360 and $1000 \mathrm{~nm}$ in response to a magnetic field slowly rotating at $f_{B}=1 \mathrm{~Hz}$ in the plane of incidence. The dominant frequency component of the resulting optical modulation is at twice the drive frequency $2 f_{B}$ because of the two-fold rotational symmetry observed in Figure 3a. Particles that lack $\mathrm{C}_{2}$ symmetry, or have their axis of rotation misaligned with respect to the rotation axis of the magnetic field would contribute to intensity at $f_{B}$ in the discrete Fourier transform of the CD. The small component at $f_{B}$, having a strength less than $30 \mathrm{~dB}$ below the dominant harmonic, attests to the uniformity of the nanohelices, and their high degree of magnetic alignment (see Figure S11). 
Switching the magneto-plasmonic nanohelices is the basis for their use as nanorheological probes that can operate even in complex fluid environments with strong absorption and a large volume fraction of scattering microparticles. We subject the magnetic helices to a rapidly rotating magnetic field and measure the phase and amplitude of the resulting CD signal. Figure $1 \mathrm{c}$ shows a helix rotating at an angular frequency $\omega$ in response to a magnetic field rotating at frequency $f_{B}$. Viscous drag causes a phase lag $\phi$ between the rotating magnetic field and the particle's magnetic moment $\mathbf{m}$, which is fixed to its orientation. ${ }^{47}$ In the steady state, $\omega=2 \pi f_{B}$, the balance of torques means that the phase angle is related to the drive frequency according to ${ }^{48}$ (see Supporting Information Note S6 for more details)

$$
\sin \phi=\frac{2 \pi \chi}{m_{r} B} \eta f_{B}
$$

where $\chi$ is a constant describing the hydrodynamic shape of the nanohelix $\left(\chi=8 \pi R^{3}\right.$ for a sphere $)^{49}, m_{r}$ is the magnetic remanence of a single Au-Fe nanohelix, and $\eta$ is the local viscosity. The key insight is that the modulated chiroptical signal reflects the particle's alignment $\mathbf{m}$ and can therefore be used to extract the otherwise unknown phase angle $\phi$. Phase sensitive measurements of the $\mathrm{CD}$ in response to the rotating magnetic field at $\mathbf{B}=3$ $\mathrm{mT}$ are shown in Figure 4a (see also Figure S12). For this only one operating wavelength is necessary so that we have selected the wavelength at $\lambda=880 \mathrm{~nm}$, where the optical density is maximal among the target systems used for nanorheology in this Letter (e.g. Figure 5a). At higher frequencies $(>50 \mathrm{~Hz})$ the viscous drag exceeds the magnetic torque and the nanohelices no longer rotate synchronously, but in the synchronous regime $\sin \phi$ is proportional to the frequency. As shown in Figure $4 \mathrm{~b}$, the slope $d \sin \phi / d f_{B}$ is itself linearly dependent on the dynamic viscosity of the medium (See Figure S13 for the results under different viscous conditions). So by calibrating the response in a series of glycerol-water mixtures with known viscosities we can recover the value for the sole free parameter, the shape constant $\chi=$ $1.35 \times 10^{7} \mathrm{~nm}^{3}$ (see Supporting Information Note S6 for comparison with calculated values). 
This process allows the nanohelices to be used as calibrated probes of their local rheological environment.

Using the same experimental procedure, we measured the viscosities of complex fluids composed of different volume fractions of $8 \mu \mathrm{m}$ polystyrene microspheres with a fluid phase of water or $20 \%$ glycerol-water. Adding the microspheres produces a slurry and leads to a dramatic increase in the sample's optical density, as seen in the left panel in Figure 4c, which rises from below OD 0.5 to OD $\sim 2.5$ ( $<1 \%$ light transmittance) (see also Figure S14). Sample opacities this high normally make optical measurements challenging. Nevertheless, the CD response from the magneto-plasmonic nanohelices is still apparent (see right panel in Figure 4c). The measured nanoscale viscosity (Figure 4d) shows no substantial change in response to the increase in the microsphere volume fraction $v_{\mathrm{ps}}$ but clearly distinguishes the differences in fluid phase viscosity between the water (red) and 20\% glycerol (blue) samples. Figure $4 \mathrm{e}$ shows that the measured relative dynamic viscosity $\eta_{r}=\frac{\eta}{\eta_{0}}$, where $\eta_{0}$ is the viscosity of the fluid phase, is essentially independent of the volume fraction of microspheres. On the other hand, the macroscale viscosity, characterized by the Krieger-Dougherty model, is over 10 times higher at a volume fraction of $50 \%{ }^{21}$. The difference arises because macroscopic rheometry measures the complex viscoelastic contributions of the microspheres, whereas the nanohelices only measure the unchanging nanoscale viscosity of the interstitial fluid. Thus, nanohelices are able to measure the nanoscale mechanical properties of a matrix phase even in the presence of aggregates that strongly affect the optical extinction and macroscale rheology.

This sensing scheme can be extended to biological fluids. Blood is a particularly challenging test case as it strongly absorbs light ${ }^{50}$. However, we conducted experiments on isolated blood plasma, and blood plasma reconstituted with four different hematocrit concentrations (blood cell volume fractions). Again, adding red blood cells (erythrocytes) leads to a significant increase in the sample's optical density (up to OD 3, see left panel in 
Figure 5a), but the CD response from the helices is still measureable through whole blood in the spectral range from $\sim 600 \mathrm{~nm}$ to $\sim 1,100 \mathrm{~nm}$ (right panel in Figure 5a). One important consideration when using nanoparticles in real bio-fluids is the formation of protein coronas. These diffuse shells of proteins aggregated around the nanoparticle can affect its dynamics ${ }^{51 \text {, }}$ 52. To minimize differences between samples of different hematocrit levels, we adopted a standardized procedure whereby the nanoparticles were mixed in pure blood plasma to develop the corona, before introducing the erythrocytes and then quickly performing the nanorheological measurement (Figure S15). Control tests in phosphate buffer saline indicate that the colloids are stable over timescales longer than the $5 \mathrm{~min}$ it takes to make a rheological measurement (see Supplementary Fig. S16). Figure 5b shows the observed blood viscosities as a function of hematocrit concentration. Clear differences are apparent between the macrorheological measurements (blue and green), which rise with hematocrit percentage, and the nano-rheological measurements (red), which show a low and constant viscosity (e.g. $p<$ 0.001 at 50\% hematocrit, see also Figures S17-18). Such measurements therefore represent an novel means for observing blood plasma viscosity, an important indicator in the diagnosis of chronic disorders, malignancy, vascular diseases, hematological cancers, and cardiovascular diseases $^{22,23}$, without the need to first separate blood cells from whole blood.

We have demonstrated that chiral magneto-plasmonic particles can be used for nanorheological measurements. The combination of a magnetic and a plasmonic material in a chiral shape gives rise to distinct optical spectra that are a function of the nanocolloids' orientation in solution. A rotating magnetic field was used to actuate the nanocolloids and produce a modulated optical response. Such dynamically controllable chiroptical nanostructures have potential in their own right as nanoscale sensors, switches ${ }^{18,19,43,53}$ and probes of higher order quadrupolar interaction terms that would normally be undetectable because of orientational averaging ${ }^{54}$. Here we have demonstrated that the chiral nanohelices 
function as wireless mechanical nanosensors capable of selectively measuring a fluid's viscosity even in the presence of scatterers and strong absorption, e.g. blood cells $(50 \%$ volume fraction and $\mathrm{OD} \sim 3$ ). The presence of microparticles and cells can dominate any macroscopic rheological measurement, but they do not directly affect a nanorheological probe. Such optical (plasmonic) sensing methods could be appealing for medical applications, as they allow for non-invasive measurements. We therefore see 'in situ active nanorheology' as a promising candidate for the measurement of viscosity in complex biological fluids, such as blood plasma ${ }^{55}$ in whole blood, with only microscopic sample volumes required. We also believe that this scheme can be applied to fluids with non-Newtonian properties ${ }^{47}$, this would expand its breadth to include measurement of the nanoscale mechanics of biomaterials such as the intracellular matrix. 

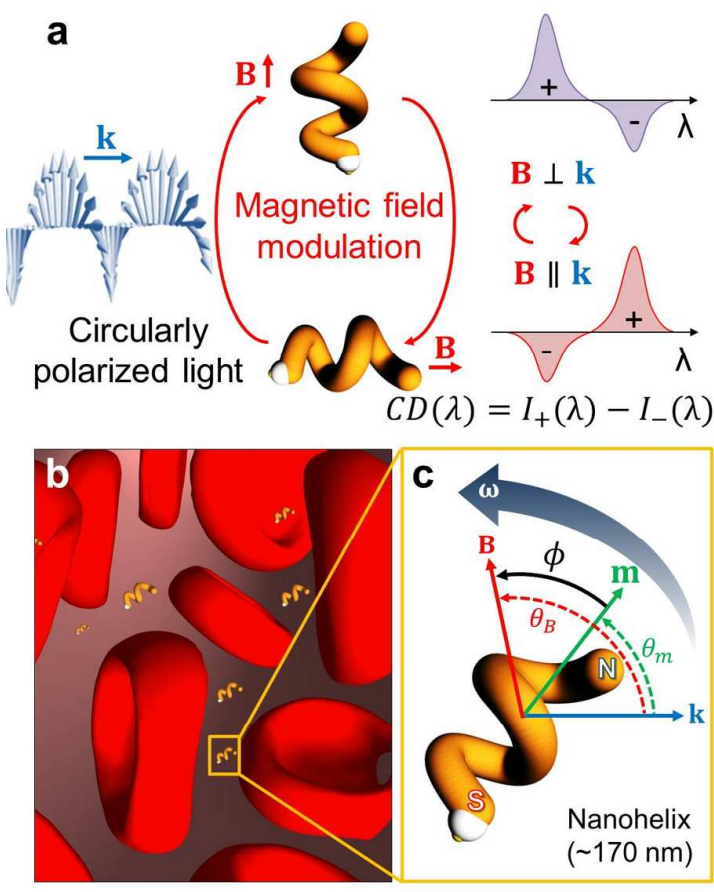

Figure 1. In situ active nanorheology with magneto-plasmonic enantiomorphs. (a) Principle of the magnetically driven chiroptical switching. Under the application of an external magnetic field $\mathbf{B}$ the ferromagnetic nanostructures align, and the $\mathrm{CD}$ response of a solution of chiral nanocolloids is modulated. (b) Schematic depicting a rotating magneto-plasmonic nanoprobe in a complex and optically dense medium (here blood plasma surrounded by erythrocytes). (c) Definition of the angles and parameters used in the nanorheological experiments (B: applied magnetic field, $\mathbf{m}$ : magnetic moment along the long axis of the nanohelix, $\mathbf{k}$ : wave vector of the incident light, $\phi$ : phase lag between $\mathbf{B}$ and $\mathbf{k}, \theta_{B}$ : angle between $\mathbf{B}$ and $\mathbf{k}, \theta_{m}$ : angle between $\mathbf{m}$ and $\mathbf{k}, \omega$ : angular velocity of the nanohelix). 

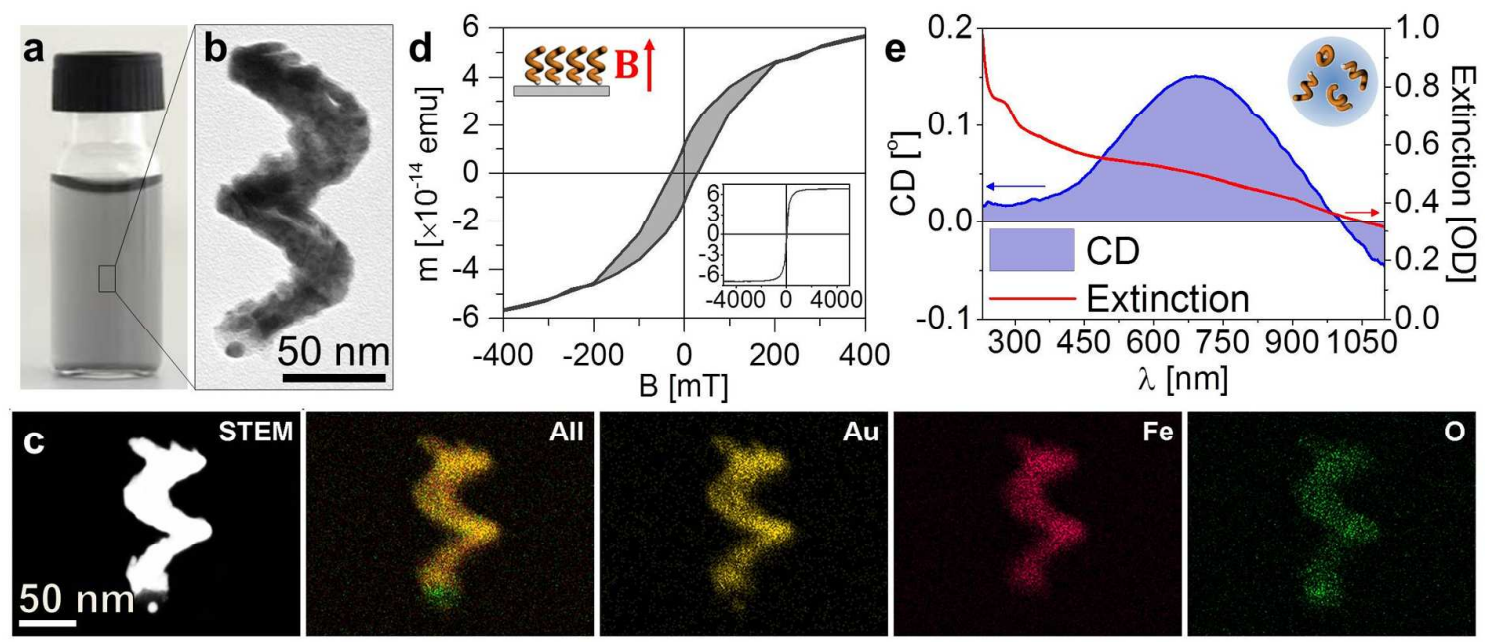

Figure 2. Characterization of magneto-plasmonic enantiomorphs. (a) A colloidal suspension of $\mathrm{Au}-\mathrm{Fe}$ nanohelices after sonication. (b) a bright-field transmission electron microscopy (TEM) image, (c) an scanning TEM (STEM) image and serial energy dispersive x-ray spectroscopy (EDX) false-color elemental map of the 2-turn Au-Fe nanohelix $\sim 170 \mathrm{~nm}$ in height. (d) A magnetic hysteresis curve of an array of the Au-Fe nanohelices measured on a Si substrate with a static magnetic field along the long axis of the nanohelices, ranging from $400 \mathrm{mT}$ to $400 \mathrm{mT}$ (coercive field: $\sim 29.5 \mathrm{mT}$ ). The inset shows the full hysteresis curve ranging from $-5 \mathrm{~T}$ to $5 \mathrm{~T}$. (e) $\mathrm{CD}$ spectrum (blue line and left $y$-axis) and UV-Vis-NIR spectrum (red line and right $\mathrm{y}$-axis) of the colloidal $\mathrm{Au}-\mathrm{Fe}$ nanohelices at $\mathbf{B}=0$ (isotropic state). 


\section{a}
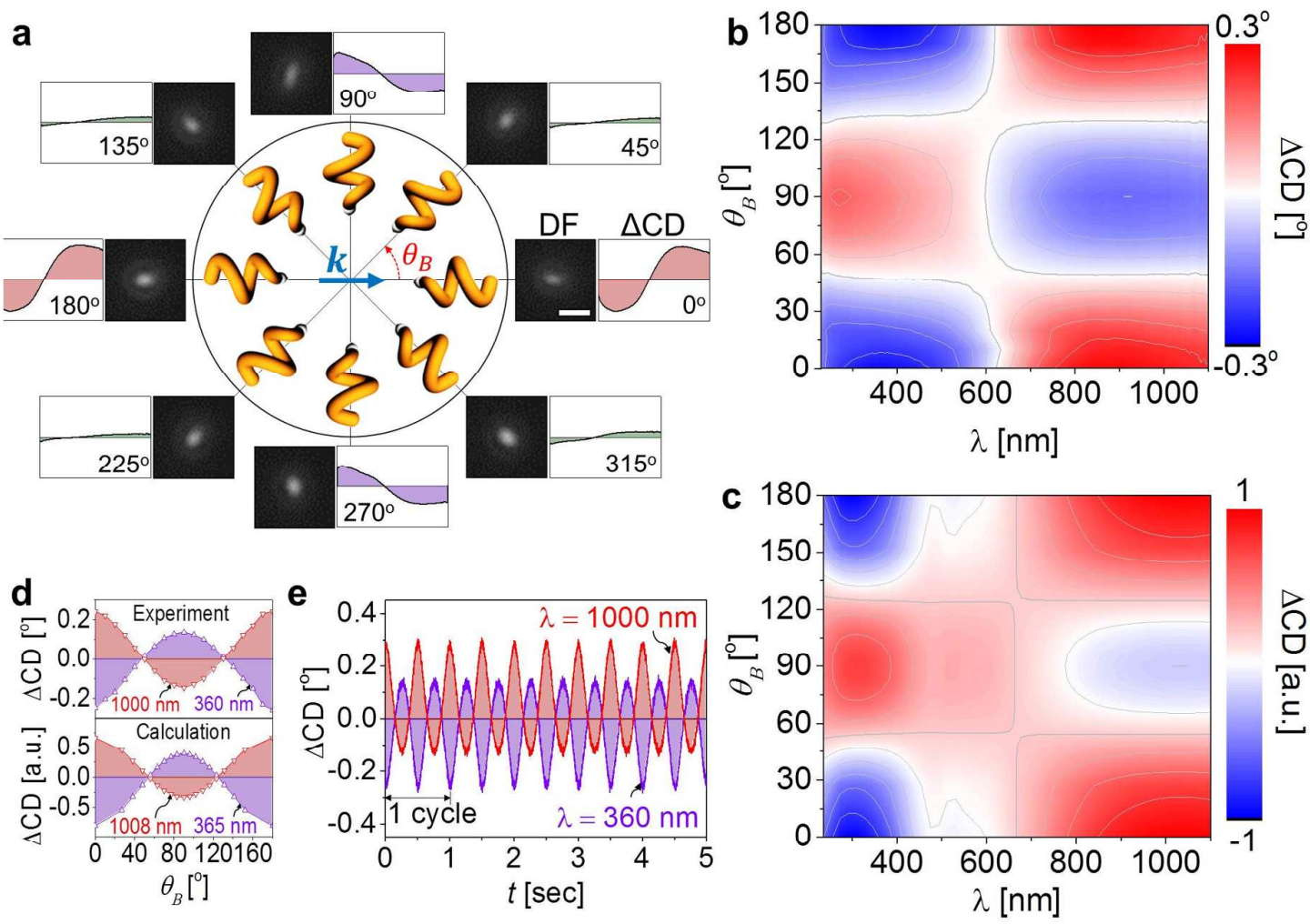

Figure 3. Magnetically driven chiroptical switching of the colloidal Au-Fe nanohelices. (a) $\Delta \mathrm{CD}$ responses of the $\mathrm{Au}-\mathrm{Fe}$ nanohelices as a function of $\lambda$ under a static magnetic field $(\mathbf{B}=$ $1 \mathrm{mT})$ at $45^{\circ}$ angular intervals. The spectra show a wavelength range of $230-1,100 \mathrm{~nm}$ on the $\mathrm{x}$-axis, and $\triangle \mathrm{CD}$ from $-0.3^{\circ}$ to $0.3^{\circ}$ on the y-axis. The dark-field images of a single $\mathrm{Au}-\mathrm{Fe}$ nanohelix (scale bar: $1 \mu \mathrm{m}$ ) are snapshots taken during continuous in-plane rotation and are shown next to the corresponding $\triangle \mathrm{CD}$ spectra from an ensemble of particles, (See Supporting Information Video 3). 2D maps of (b) experimental and (c) theoretical $\Delta \mathrm{CD}$ responses of the $\mathrm{Au}-\mathrm{Fe}$ nanohelices as functions of $\lambda$ (x-axis) and $\theta_{B}$ (y-axis). (d) $\Delta \mathrm{CD}$ response of the colloidal Au-Fe nanohelices as a function of $\theta_{B}$ (upper plots: experimental results at two wavelengths $\lambda=360 \mathrm{~nm}$ (violet) and $\lambda=1,000 \mathrm{~nm}$ (red), lower plots: theoretical results at $\lambda=$ $365 \mathrm{~nm}$ (violet) and $\lambda=1,008 \mathrm{~nm}$ (red)). (e) $\Delta \mathrm{CD}$ response of the colloidal Au-Fe nanohelices at $\lambda=360 \mathrm{~nm}$ (violet) and $\lambda=1,000 \mathrm{~nm}$ (red) recorded for a rotating field $\mathbf{B}$ with $f_{B}=1 \mathrm{~Hz}$, showing stable signals under continuous switching. 

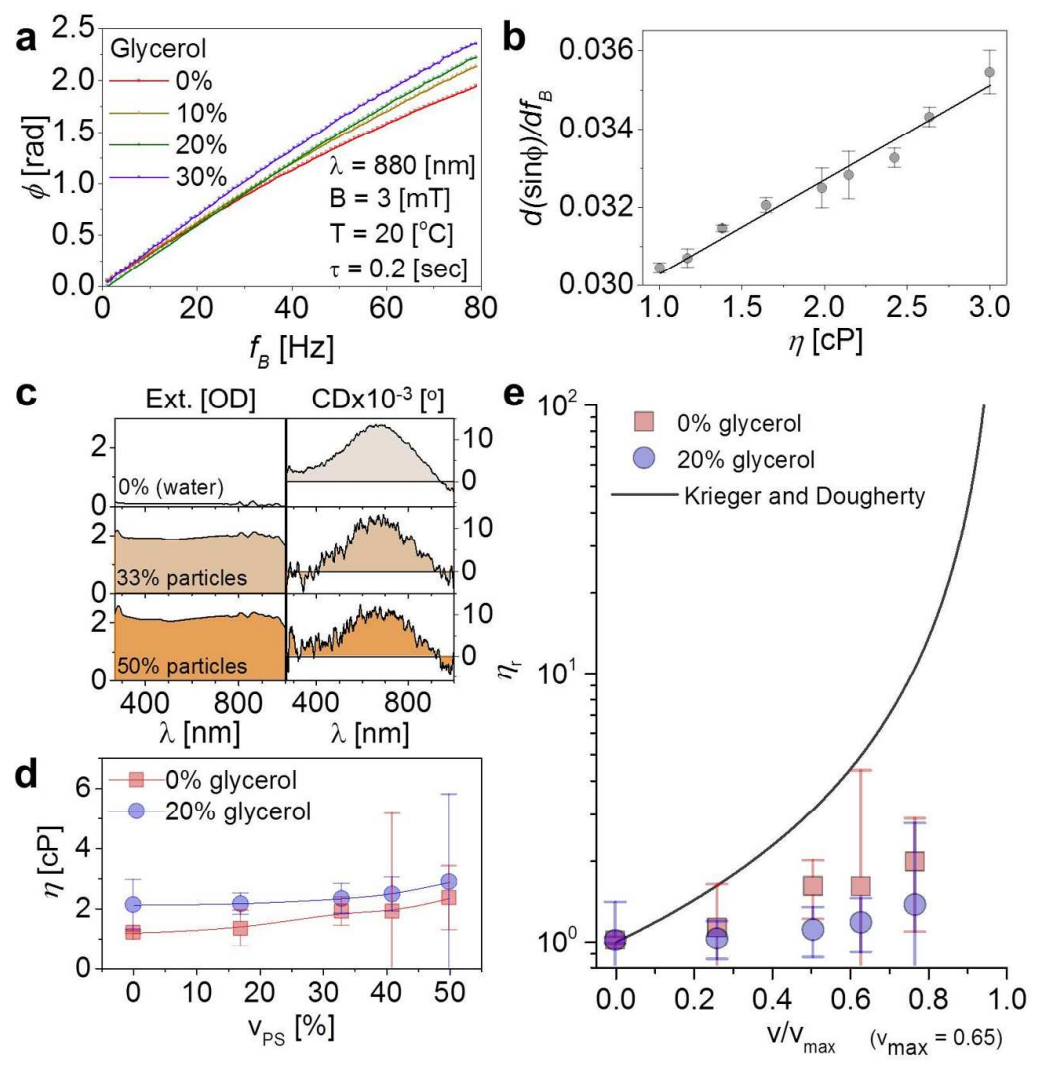

Figure 4. In situ active nanorheology. (a) Phase angle, $\phi$ of the colloidal Au-Fe nanohelices in the presence of different concentrations of a glycerol-water mixture as a function of $f_{B}$ (at $\mathbf{B}=3 \mathrm{mT}, \lambda=880 \mathrm{~nm}$ at $\mathrm{T}=20^{\circ} \mathrm{C}$ ). (b) The corresponding slopes, $d \sin \phi / d f_{B}$, as a function of the dynamic viscosity of the medium, $\eta$. (c) Extinction (left panel) and CD (right panel) spectra of the colloidal Au-Fe nanohelices in water in the presence of polystyrene particles ( 8 $\mu \mathrm{m}$ in diameter) with different volume fractions (top: $0 \%$, middle: $33 \%$, bottom: $50 \%$ ). The extinction spectra are featureless, whereas a clear CD peak is discernible even at high particle fraction. (d) Dynamic viscosity as a function of the volume fraction of the polystyrene microspheres in water (red squares) and 20\% glycerol (blue circles). (e) The corresponding relative dynamic viscosities, $\eta_{r}$ at the nanoscale (red squares and blue circles) and at the macro scale (solid line) $)^{21,56}$. 

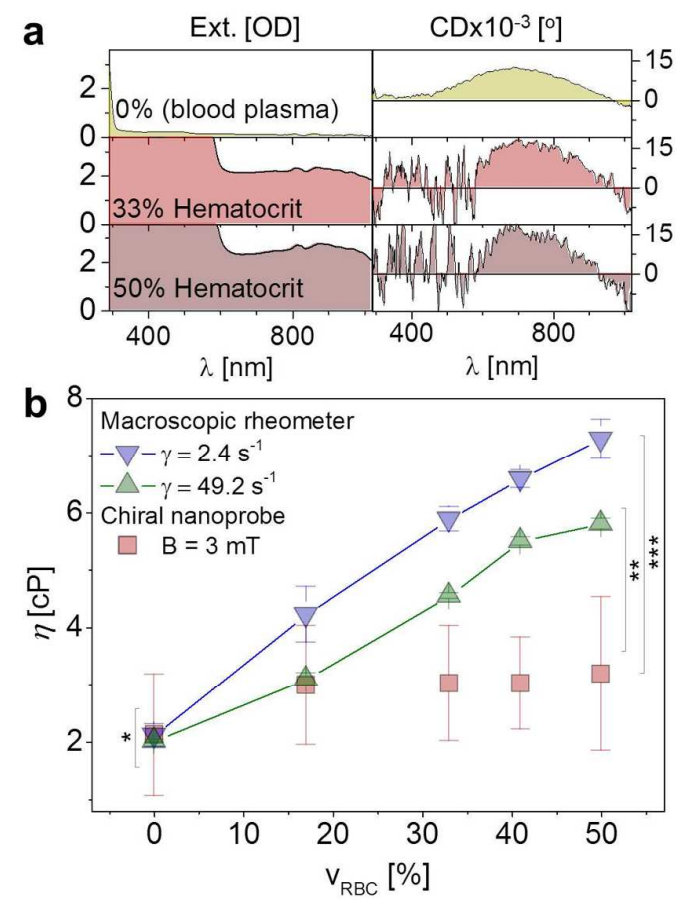

Figure 5. In situ active nanorheology in blood. (a) Extinction and CD spectra of the colloidal $\mathrm{Au}-\mathrm{Fe}$ nanohelices as a function of $\lambda$ in bovine blood plasma surrounded by the erythrocytes at different volume fractions (top: $0 \%$, middle: 33\%, bottom: 50\%). (b) The measured dynamic viscosity, $\eta$ of the bovine blood plasma in the presence of red blood cells with different hematocrit level. The macroscopic measurements were made at different shear rates (green top triangles: $2.4 \mathrm{~s}^{-1}$ and blue bottom triangle: $49.2 \mathrm{~s}^{-1}$ ). The star symbols $(*)$ represent the statistical analysis of the measured viscosities at $0 \%$ and $50 \%$ hematocrit (rheometer: $n=3$, nanoprobe: $\mathrm{n}=4) .{ }^{*} p>0.1, * * p<0.01$, and ${ }^{* *} p<0.001$ (one-way ANOVA). 


\section{METHODS}

Block copolymer micelle nanolithography (BCML): The hexagonal array of $\mathrm{Au}$ nanoparticles was prepared using block-copolymer micelle nanolithography as previously reported $^{38}$. Poly (styrene)-b-poly (2-vinylpyridine) (S units: 1056; VP units: 671) was dissolved in toluene at a concentration of $4 \mathrm{mg} \cdot \mathrm{mL}^{-1}$ and stirred overnight. $\mathrm{HAuCl}_{4} \cdot 3\left(\mathrm{H}_{2} \mathrm{O}\right)$ was added to the polymer solution at a molar ratio of 0.5 per vinyl pyridine unit and stirred for at least 48 hrs. Spherical micelles, loaded with Au salts in the core, form by self-assembly. The micelles are spin-coated onto a two-inch Si wafer at $8000 \mathrm{rpm}$ for $1 \mathrm{~min}$ where they form a quasi-hexagonally close-packed monolayer. Subsequent plasma treatment under $10 \% \mathrm{H}_{2}$ and 90\% Ar plasma (power: $350 \mathrm{~W}$, pressure: $0.4 \mathrm{mbar}$, time: $45 \mathrm{~min}$ ) reduces the Au to form nanoparticles with $\sim 12 \mathrm{~nm}$ in diameter and spaced $\sim 100 \mathrm{~nm}$ apart (as measured by scanning electron microscopy (SEM)).

Nano Glancing angle deposition (nanoGLAD): Au-Fe nanohelices were grown on the array of the Au nanoparticles in a custom-built GLAD system at $\mathrm{T}=90 \mathrm{~K}$, based on codeposition from two electron-beam evaporators with a base pressure of $1 \times 10^{-6} \mathrm{mbar}$. The substrate manipulator provides independent control over the azimuthal direction $\varphi$, and molecular flux direction $\alpha$ during deposition. To grow nanohelices, the flux angle $\alpha$ and the azimuthal rotation rate per unit thickness $d \varphi / d w$ were kept to respectively $87^{\circ}$ and $1.8 \pm 0.1$ $\mathrm{o} / \mathrm{nm}$ with closed-loop feedback based on measurements of material deposition rates on a quartz crystal monitor. The alloy stoichiometry was controlled by measuring and controlling the deposition rates from each evaporator independently to maintain the desired ratio.

Preparation of colloidal solution: The grown nanohelices were removed from the wafer by bath sonication of a wafer piece in an aqueous solution of $1 \mathrm{mM}$ sodium citrate for $\sim 2 \mathrm{~min}$. 
SEM, TEM, and STEM-EDX analysis: Structures were imaged with SEM (Gemini ultra 55, Zeiss), TEM (CM200, Philips), and STEM (SESAM, Zeiss). TEM samples were prepared by drop casting $\sim 10 \mu \mathrm{L}$ of the colloidal solution onto a Holey ${ }^{\circledR}$ carbon coated TEM grid $(\mathrm{Cu}$ 400 mesh), followed by drying under a gentle flow of Ar gas. Bright-field TEM images were recorded under an accelerating voltage of $200 \mathrm{kV}$.

UV-VIS-NIR spectroscopy and CD spectroscopy: Extinction spectra of the nanocolloids were measured on a Cary UV-VIS-NIR 5000 spectrophotometer. Circular dichroism spectra were obtained with a Jasco J-810 circular dichroism spectrometer. All the spectra were measured with a scan rate of $500 \mathrm{~nm} \cdot \mathrm{min}^{-1}$ in the wavelength range of $\sim 300$ to $1050 \mathrm{~nm}$ with $0.1 \mathrm{~nm}$ intervals.

Dark-field optical microscopy: Dark field microscopy videos were taken on a Zeiss Axio Observer Z1 inverted microscope with an attached Basler acA2000-340kc camera. Videos were taken at a frame rate of $20 \mathrm{f} \cdot \mathrm{sec}^{-1}$. A square, double-sided piece of tape with the center removed was adhered on a clean glass cover slip to create a fluid chamber $(\sim 20 \mu \mathrm{L})$ and the colloidal solution was sandwiched between two cover slips. A $100 \times$ objective lens $(\mathrm{NA}=$ 1.25 , oil immersion) and a dark filed condenser $(\mathrm{NA}=1.2 / 1.4)$ were used to collect the light scattered from the Au-Fe nanohelices.

SQUID magnetometer: SQUID measurements were taken of the array of Au-Fe nanohelices on a piece of Si wafer $\left(\sim 0.5 \mathrm{~cm}^{2}\right)$ out of plane (OOP) at $300 \mathrm{~K}$ using a Quantum Design magnetic property measurement system (MPMS) magnetometer.

Numerical simulations: The chiroptical responses of $\mathrm{Au}-\mathrm{Fe}$ nanohelices for the alignment of $\theta_{B}=0^{\circ}$ to $180^{\circ}$ were numerically calculated using the DDSCAT implementation of the discrete dipole approximation ${ }^{57}$. Calculations were performed over wavelengths between 200 
$\mathrm{nm}$ and $1100 \mathrm{~nm}$ with $18 \mathrm{~nm}$ intervals based on the calculated dielectric function of Au-Fe with an atomic ratio of 1:1.5.

Fluid and blood sample preparation: Glycerol-water mixtures were prepared at concentrations of $0-50 \%$. The viscosities were based on values found in Ref. ${ }^{58}$. Polystyrene beads of $8 \mu \mathrm{m}$ diameter suspended in water (PPS-8.0, Kiskerbiotech GmbH, Germany) were used for the particle rheology. The volume fraction of the beads was controlled with 2:10 (17\%), 5:10 (33\%), 7:10 (41\%), 10:10 (50\%) volume ratios in water. Blood plasma was separated from whole bovine blood stabilized in the anticoagulant citrate dextrose (ACD) solution (Fiebig-Nährstofftechnik, Germany) by centrifuging at $2000 \mathrm{~g}$ for $15 \mathrm{~min}$. For the nanorheology measurements, the Au-Fe nanohelices were removed from the wafer by sonicating a piece of sample wafer $\left(\sim 0.3 \mathrm{~cm}^{2}\right)$ in $1 \mathrm{~mL}$ of blood plasma for $\sim 2 \mathrm{~min}$. Samples were also prepared with hematocrit by mixing blood plasma with packed erythrocytes at 2:10 (17\%), 5:10 (33\%), 7:10 (41\%), 10:10 (50\%) volume ratios. A rotational rheometer (HAAKE RheoStress 1, Thermo Scientific) with a parallel plate geometry was used to measure the viscosity, and $\sim 40 \mathrm{~mL}$ of blood sample was measured at shear rates ranging from 2 to $100 \mathrm{~s}^{-1}$ (at a solution temperature of $20^{\circ} \mathrm{C}$ ).

Nanorheological viscosity measurements: Suspended magnetic nanohelices were subjected to rotating magnetic fields over a range of frequencies $f_{B}=1$ to $80 \mathrm{~Hz}$ at $\mathbf{B}=3 \mathrm{mT}$ at $\mathrm{T}=20{ }^{\circ} \mathrm{C}$. The amplitude and the phase angle of the $\mathrm{CD}$ response at $\lambda=880 \mathrm{~nm}$ were measured using a lock-in amplifier referenced to the magnetic field modulation. The phase was carefully corrected for phase lags introduced by the frequency dependent inductance of the magnetic coils, and the mechanical phase angle was recovered from the optical phase. Small volume cuvettes $(\sim 300 \mu \mathrm{L})$ with $1 \mathrm{~mm}$ path lengths were used. 


\title{
ASSOCIATED CONTENT
}

Supporting Information: This material is available free of charge via the Internet at http://pubs.acs.org.

\section{AUTHOR INFORMATION}

Corresponding Author: *fischer@,is.mpg.de

\begin{abstract}
Author Contributions: H.-H.J., A.G.M., T.-C.L, J.G.G and P.F. designed the experiments. H.-H.J. and J.G.G performed the CD and dark-field experiments. T.-C.L and M.A.-C. carried out the TEM imaging. S.E. and A.G.M conducted the CD calculation. H.-H.J. and A.G.M. developed the experimental setup and measured and analyzed the data. T.Q. performed the blood macrorheological measurements, and H.-H.J., A.G.M., T.-C.L. and P.F. wrote the paper.
\end{abstract}

Notes: The authors declare no competing financial interests.

\section{ACKNOWLEDGMENT}

We are grateful to C. Miksch and J. P. Spatz for providing us with BCML substrates and for SEM access. We thank G. Schütz for SQUID access, the ZWE Analytical Chemistry for the ICP-OES analysis, B. Siegle and D. Flötotto for the AES measurement, and the Stuttgart Centre for Electron Microscopy for technical support with the TEM and STEM imaging. We are also grateful to D. Walker, J. Kim, and K. Son for helpful comments. This work was supported by the Max Planck-EPFL center for molecular nanoscience and technology, and the European Research Council under the ERC Grant agreement 278213. 


\section{REFERENCES}

1. Mayer, K. M.; Hafner, J. H. Chemical Reviews 2011, 111, (6), 3828-3857.

2. Atwater, H. A.; Polman, A. Nat Mater 2010, 9, (3), 205-213.

3. Brongersma, M. L.; Halas, N. J.; Nordlander, P. Nat Nano 2015, 10, (1), 25-34.

4. Prodan, E.; Radloff, C.; Halas, N. J.; Nordlander, P. Science 2003, 302, (5644), 419-422.

5. Motl, N. E.; Smith, A. F.; DeSantis, C. J.; Skrabalak, S. E. Chemical Society Reviews 2014, 43, (11), 3823-3834.

6. Ding, T.; Sigle, D.; Zhang, L.; Mertens, J.; de Nijs, B.; Baumberg, J. ACS Nano 2015, 9, (6), 61106118.

7. Jeong, H.-H.; Mark, A. G.; Alarcon-Correa, M.; Kim, I.; Oswald, P.; Lee, T.-C.; Fischer, P. Nat Commun 2016, 7, 11331, 10.1038/ncomms11331.

8. Armelles, G.; Cebollada, A.; García-Martín, A.; González, M. U. Advanced Optical Materials 2013, 1, (1), 10-35.

9. Valev, V. K.; Baumberg, J. J.; Sibilia, C.; Verbiest, T. Advanced Materials 2013, 25, (18), 25172534.

10.Sheldon, M. T.; van de Groep, J.; Brown, A. M.; Polman, A.; Atwater, H. A. Science 2014, 346, (6211), 828-831.

11.Kuzyk, A.; Schreiber, R.; Fan, Z.; Pardatscher, G.; Roller, E.-M.; Hogele, A.; Simmel, F. C.; Govorov, A. O.; Liedl, T. Nature 2012, 483, (7389), 311-314.

12.Mark, A. G.; Gibbs, J. G.; Lee, T.-C.; Fischer, P. Nat Mater 2013, 12, (9), 802-807.

13.Ogier, R.; Fang, Y.; Svedendahl, M.; Johansson, P.; Käll, M. ACS Photonics 2014, 1, (10), 10741081.

14.Frank, B.; Yin, X.; Schäferling, M.; Zhao, J.; Hein, S. M.; Braun, P. V.; Giessen, H. ACS Nano 2013, 7, (7), 6321-6329.

15.McPeak, K. M.; van Engers, C. D.; Blome, M.; Park, J. H.; Burger, S.; Gosálvez, M. A.; Faridi, A.; Ries, Y. R.; Sahu, A.; Norris, D. J. Nano Letters 2014, 14, (5), 2934-2940.

16.Gibbs, J. G.; Mark, A. G.; Eslami, S.; Fischer, P. Applied Physics Letters 2013, 103, (21), -

17.Fan, Z.; Govorov, A. O. Nano Letters 2012, 12, (6), 3283-3289.

18.Zhao, Y.; Xu, L.; Ma, W.; Wang, L.; Kuang, H.; Xu, C.; Kotov, N. A. Nano Letters 2014, 14, (7), 3908-3913.

19.HendryE; CarpyT; JohnstonJ; PoplandM; Mikhaylovskiy, R. V.; Lapthorn, A. J.; Kelly, S. M.; Barron, L. D.; GadegaardN; KadodwalaM. Nat Nano 2010, 5, (11), 783-787.

20.Fung, Y., Biomechanics: mechanical properties of living tissues. Springer-Verlag: 1981.

21.Krieger, I. M.; Dougherty, T. J. Transactions of The Society of Rheology 1959, 3, (1), 137-152.

22.Kersaudy-Kerhoas, M.; Sollier, E. Lab on a Chip 2013, 13, (17), 3323-3346.

23.Késmárky, G.; Kenyeres, P.; Rábai, M.; Tóth, K. Clinical Hemorheology \& Microcirculation 2008, $39,(1 / 4), 243-246$.

24.Windberger, U.; Bartholovitsch, A.; Plasenzotti, R.; Korak, K. J.; Heinze, G. Experimental Physiology 2003, 88, (3), 431-440.

25.Fedosov, D. A.; Pan, W.; Caswell, B.; Gompper, G.; Karniadakis, G. E. Proceedings of the National Academy of Sciences 2011, 108, (29), 11772-11777.

26.Chang, W.-S.; Lassiter, J. B.; Swanglap, P.; Sobhani, H.; Khatua, S.; Nordlander, P.; Halas, N. J.; Link, S. Nano Letters 2012, 12, (9), 4977-4982.

27.Zhang, S.; Zhou, J.; Park, Y.-S.; Rho, J.; Singh, R.; Nam, S.; Azad, A. K.; Chen, H.-T.; Yin, X.; Taylor, A. J.; Zhang, X. Nat Commun 2012, 3, 942, 10.1038/ncomms190.

28.Chen, H.-T.; Padilla, W. J.; Zide, J. M. O.; Gossard, A. C.; Taylor, A. J.; Averitt, R. D. Nature 2006, 444, (7119), 597-600.

29.Zhu, B.; Feng, Y.; Zhao, J.; Huang, C.; Jiang, T. Applied Physics Letters 2010, 97, (5), -.

30.van der Zande, B. M. I.; Koper, G. J. M.; Lekkerkerker, H. N. W. The Journal of Physical Chemistry B 1999, 103, (28), 5754-5760.

31.Sönnichsen, C.; Alivisatos, A. P. Nano Letters 2004, 5, (2), 301-304.

32.Khatua, S.; Chang, W.-S.; Swanglap, P.; Olson, J.; Link, S. Nano Letters 2011, 11, (9), 3797-3802.

33.Wang, M.; Gao, C.; He, L.; Lu, Q.; Zhang, J.; Tang, C.; Zorba, S.; Yin, Y. Journal of the American Chemical Society 2013, 135, (41), 15302-15305.

34.Liu, Q.; Cui, Y.; Gardner, D.; Li, X.; He, S.; Smalyukh, I. I. Nano Letters 2010, 10, (4), 1347-1353. 
35.Schamel, D.; Mark, A. G.; Gibbs, J. G.; Miksch, C.; Morozov, K. I.; Leshansky, A. M.; Fischer, P. ACS Nano 2014, 8(9), 8794-8801.

36.Tuteja, A.; Mackay, M. E.; Narayanan, S.; Asokan, S.; Wong, M. S. Nano Letters 2007, 7, (5), 1276-1281.

37.Chevry, L.; Sampathkumar, N. K.; Cebers, A.; Berret, J. F. Physical Review E 2013, 88, (6), $062306,1-8$.

38.Glass, R.; Möller, M.; Spatz, J. P. Nanotechnology 2003, 14, (10), 1153-1160.

39.Robbie, K.; Sit, J. C.; Brett, M. J. Journal of Vacuum Science \&amp; Technology B 1998, 16, (3), $1115-1122$.

40.Chiang, I. C.; Chen, D. H. Advanced Functional Materials 2007, 17, (8), 1311-1316.

41.Habteyes, T. G.; Dhuey, S.; Wood, E.; Gargas, D.; Cabrini, S.; Schuck, P. J.; Alivisatos, A. P.; Leone, S. R. ACS Nano 2012, 6, (6), 5702-5709.

42.Schreiber, R.; Luong, N.; Fan, Z.; Kuzyk, A.; Nickels, P. C.; Zhang, T.; Smith, D. M.; Yurke, B.; Kuang, W.; Govorov, A. O.; Liedl, T. Nat Commun 2013, 4, 3948, 10.1038/ncomms3948.

43.Kuzyk, A.; Schreiber, R.; Zhang, H.; Govorov, A. O.; Liedl, T.; Liu, N. Nat Mater 2014, 13, (9), 862-866.

44.Zhou, C.; Duan, X.; Liu, N. Nat Commun 2015, 6, 8102, 10.1038/ncomms9102.

45. Claborn, K.; Isborn, C.; Kaminsky, W.; Kahr, B. Angewandte Chemie International Edition 2008, 47, (31), 5706-5717.

46.Phatak, C.; Liu, Y.; Gulsoy, E. B.; Schmidt, D.; Franke-Schubert, E.; Petford-Long, A. Nano Letters 2014, 14, (2), 759-764.

47.Berret, J. F. Nat Commun 2016, 7, 10134, 10.1038/ncomms10134.

48.Schamel, D.; Pfeifer, M.; Gibbs, J. G.; Miksch, B.; Mark, A. G.; Fischer, P. Journal of the American Chemical Society 2013, 135, (33), 12353-12359.

49.Eric, L.; Thomas, R. P. Reports on Progress in Physics 2009, 72, (9), 096601, 1-36.

50.D. Howes, P.; Rana, S.; M. Stevens, M. Chemical Society Reviews 2014, 43, (11), 3835-3853.

51.Tenzer, S.; Docter, D.; Kuharev, J.; Musyanovych, A.; Fetz, V.; Hecht, R.; Schlenk, F.; Fischer, D.; Kiouptsi, K.; Reinhardt, C.; Landfester, K.; Schild, H.; Maskos, M.; Knauer, S. K.; Stauber, R. H. Nat Nano 2013, 8, (10), 772-781.

52.Pino, P. d.; Pelaz, B.; Zhang, Q.; Maffre, P.; Nienhaus, G. U.; Parak, W. J. Materials Horizons 2014, 1, (3), 301-313.

53.Wei, Q.; Song, H.-M.; Leonov, A. P.; Hale, J. A.; Oh, D.; Ong, Q. K.; Ritchie, K.; Wei, A. Journal of the American Chemical Society 2009, 131, (28), 9728-9734.

54.Buckingham, A. D.; Dunn, M. B. Journal of the Chemical Society A: Inorganic, Physical, Theoretical 1971, (0), 1988-1991.

55.Brust, M.; Schaefer, C.; Doerr, R.; Pan, L.; Garcia, M.; Arratia, P. E.; Wagner, C. Physical Review Letters 2013, 110, (7), 078305, 1-5.

56.Einstein, A. Annalen der Physik 1906, 324, (2), 289-306.

57.Draine, B. T.; Flatau, P. J. J. Opt. Soc. Am. A 1994, 11, (4), 1491-1499.

58.Cheng, N.-S. Industrial \& Engineering Chemistry Research 2008, 47, (9), 3285-3288. 